\title{
CONTINUOUSLY HOMOGENEOUS CONTINUA AND THEIR ARC COMPONENTS
}

\author{
JANUSZ R. PRAJS
}

(Communicated by Doug W. Curtis)

\begin{abstract}
Let $X$ be a continuously homogeneous Hausdorff continuum. We prove that if there is a sequence $A_{1}, A_{2}, \ldots$ of its arc components with $X=\operatorname{cl} A_{1} \cup \operatorname{cl} A_{2}$ $\cup \cdots$, and there is an arc component of $X$ with nonempty interior, then $X$ is arcwise connected. As consequences and applications we get: (1) if $X$ is the countable union of arcwise connected continua, then $X$ is arcwise connected; (2) if $X$ is nondegenerate and metric, the number of its arc components is countable and it contains no simple triod, then it is either an arc or a simple closed curve; and, in particular, (3) an arc is the only nondegenerate continuously homogeneous arc-like metric continuum with countably many arc components.
\end{abstract}

Introduction. Recall that a space $X$ is said to be continuously homogeneous if for every two points $x, y \in X$ there is a continuous surjection $f: X \rightarrow X$ with $f(x)=y$. This notion is due to D. P. Bellamy, and also, in a more general version, to $\mathbf{J}$. J. Charatonik (see $[\mathbf{C}]$ ). The purpose of this paper is to prove that

if a Hausdorff continuum $X$ is continuously homogeneous and there is a sequence $A_{1}, A_{2}, \ldots$ of its arc components such that $X=\mathrm{cl} A_{1} \cup \mathrm{cl} A_{2} \cup \cdots$, and there is an arc component of $X$ with nonempty interior, then $X$ is arcwise connected.

As can be seen, this fact is a strengthening of the result 3 of [K2, p. 270]. P. Krupski suggested there that such an improvement (in a somewhat weaker version-see Remark in [K2, p. 271]) might be true.

Actually, conclusion (1) is one of the applications of Theorem 1 below. The notion of an arc component is replaced in this theorem by the concept of a $\mathscr{K}$-component defined as follows (compare [P1]). Let $X$ be a space and $\mathscr{K}$ be an arbitrary family of subcontinua of $X$ satisfying the two following conditions:

(i) if $K=K_{1} \cup K_{2}$ with $K_{1}, K_{2} \in \mathscr{K}$ and $K_{1} \cap K_{2} \neq \varnothing$, then $K \in \mathscr{K}$,

(ii) if $K \in \mathscr{K}$ and $f: X \rightarrow X$ is a continuous surjection, then $f(K) \in \mathscr{K}$.

A set $Y \subset X$ is said to be $\mathscr{K}$-connected if each two of its points lie in a subcontinuum of it belonging to $\mathscr{K}$. The maximal $\mathscr{K}$-connected subsets of the space

Received by the editors January 28, 1986 and, in revised form. August 6, 1986.

1980 Mathematics Subject Classification (1985 Revision). Primary 54F20; Secondary 54F65.

Key nords and phrases. Continuous homogeneity, covering sequence, Hausdorff continuum, $\mathscr{K}$-component, simple triod. 
are called its $\mathscr{K}$-components. It can easily be seen that $\mathscr{K}$-components are arc components when $\mathscr{K}$ is the family of all locally connected metric subcontinua of $X$.

Now we are ready to formulate the theorem.

1. THEOREM. If a Hausdorff continuum $X$ is continuously homogeneous and there is a sequence $A_{1}, A_{2}, \ldots$ of its $\mathscr{K}$-components such that $X=\operatorname{cl} A_{1} \cup \mathrm{cl} A_{2} \cup \cdots$, and there is a $\mathscr{K}$-component of $X$ with nonempty interior, then $X$ is $\mathscr{K}$-connected.

To give some possible applications of this theorem, other than one of (1), note that if:

$X$ is planable and $\mathscr{K}$ is the family of all $\delta$-connected subcontinua of $X$ (see [H1, $\mathbf{H} 3$ and $\mathbf{H 2}]$ ), or

$\mathscr{K}$ is the family of all metric subcontinua of $X$ with index of local disconnectivity less than $\alpha$, for $\alpha<\Omega$ (for the definition see [P2, Chapter IV]), or

$\mathscr{K}$ is the family of all weakly chainable metric subcontinua of $X$ (see [L]), or

$\mathscr{K}$ is the family of all subcontinua of $X$ that are continuous images of Hausdorff arcs,

then essentially different kinds of $\mathscr{K}$-connectedness are obtained.

In this way, i.e., by considering an arbitrary $\mathscr{K}$-connectedness instead of the arc connectedness only, we may extend a number of results concerning continuous homogeneity (e.g. Propositions 4 and 5 of [K1, p. 354], 1 of [K2, p. 269], and Theorem 3 of [CG, p. 341]).

All spaces considered here are assumed to be Hausdorff. A mapping is a continuous mapping between topological spaces, a surjection is a surjective mapping. An arc is a homeomorphic image of the unit segment [0,1], a Hausdorff arc is a linearly ordered continuum. Symbol $a b$ denotes an arc with ends $a$ and $b$. The union of three arcs $p x, p y$, and $p z$ is called a simple triod if $p x \cap p y=p x \cap p z=p y \cap$ $p z=\{p\}$. A point $e$ is called an end point of a space $X$ if $e \in X$ and for every two $\operatorname{arcs} C_{1}, C_{2} \subset X$ both containing $e$ we have either $C_{1} \subset C_{2}$ or $C_{2} \subset C_{1}$. The letters $\omega$ and $\Omega$ denote the first infinite and the first uncountable ordinal, respectively. In this paper, according to [KM, p. 235], 0 is considered to be a limit ordinal.

Covering sequences of compact spaces. The proof of Theorem 1 makes heavy use of Lemma 3 below. In order to obtain this lemma the notion of a covering sequence of a compact space is employed. This notion is analogous to the concept of a covering sequence of a metric compactum defined in [P1]. Let $X$ be a compact space with card $X \geqslant \boldsymbol{\aleph}_{0}$, and let $\Gamma$ be a limit ordinal of cardinality greater than card $X$. A sequence $\tau=\left\{X_{\alpha}\right\}_{\alpha<\Gamma}$ of compact subsets $X_{\alpha}$ of $X$ is called a covering sequence of $X$ provided for every $\alpha<\Gamma$ there is a countable ordinal $\beta$ such that $\bigcup\left\{X_{\gamma}\right.$ : $\dot{\alpha} \leqslant \gamma \leqslant \alpha+\beta\}=X$. For every covering sequence $\tau=\left\{X_{\alpha}\right\}_{\alpha<\Gamma}$ we inductively define another sequence $\left\{D_{\alpha}(\tau)\right\}_{\alpha<\Gamma}$ of compact subsets of $X$ :

$D_{0}(\tau)=X$,

$D_{\alpha+1}(\tau)=\operatorname{cl}\left(D_{\alpha}(\tau) \backslash X_{\alpha}\right)$,

$D_{\varphi}(\tau)=\bigcap\left\{D_{\alpha}(\tau): \alpha<\varphi\right\}$, for each limit ordinal $\varphi>0$. 
Note that the sequence $\left\{D_{\alpha}(\tau)\right\}_{\alpha<\Gamma}$ is decreasing. Observe the following two properties of this sequence.

For every $\alpha<\Gamma$ with $D_{\alpha}(\tau) \neq \varnothing$ there is $\beta<\Omega$ such that $D_{\alpha+\beta}(\tau) \subsetneq D_{\alpha}(\tau)$.

Indeed, let $\beta$ be a number guaranteed by the definition for the number $\alpha$. Then the family $\left\{D_{\alpha}(\tau) \cap X_{\gamma}: \alpha \leqslant \gamma \leqslant \alpha+\beta\right\}$ covers the set $D_{\alpha}(\tau)$, thus, by the Baire theorem, one of its elements $D_{\alpha}(\tau) \cap X_{\gamma_{0}}$ has nonempty interior in $D_{\alpha}(\tau)$. Therefore

$$
D_{\alpha+\beta+1}(\tau) \subset D_{\gamma_{0}+1}(\tau)=\operatorname{cl}\left(D_{\gamma_{0}}(\tau) \backslash X_{\gamma_{0}}\right) \subset \operatorname{cl}\left(D_{\alpha}(\tau) \backslash X_{\gamma_{0}}\right) \subsetneq D_{\alpha}(\tau) .
$$

There is an ordinal $\beta<\Gamma$ with $D_{\beta}(\tau)=\varnothing$.

In fact, put $P=\left\{\alpha<\Gamma: D_{\alpha+1}(\tau) \subsetneq D_{\alpha}(\tau)\right\}$. Then we have card $P \leqslant \operatorname{card} X$. By (2) we see that there are only countably many ordinals between any $\alpha \in P$ and its successor in $P$. Therefore cardinality of the ordinal sup $P$ cannot exceed the cardinal $\boldsymbol{\aleph}_{0} \cdot$ card $P \leqslant \boldsymbol{\aleph}_{0} \cdot \operatorname{card} X=$ card $X$. By the assumption about the cardinality of $\Gamma$ we have $\sup P<\Gamma$ which means that the sets $D_{\alpha}(\tau)$ for $\alpha>\sup P$ are identical. Thus $D_{\alpha}(\tau)=\varnothing$ for such $\alpha$ by (2).

The minimum ordinal $\beta$ such that $D_{\beta}(\tau)=\varnothing$ is denoted by $\lambda(\tau)$.

For further purposes we define a binary operation $*$ between covering sequences of the same space. Let $\tau=\left\{X_{\alpha}\right\}_{\alpha<\Gamma}, \psi=\left\{Y_{\alpha}\right\}_{\alpha<\Gamma}$ be covering sequences of $X$. Then we put $\tau * \psi=\left\{Z_{\alpha}\right\}_{\alpha<\Gamma}$, where

$$
Z_{2 \alpha}=X_{\alpha}, \quad Z_{2 \alpha+1}=Y_{\alpha} .
$$

Obviously $\tau * \psi$ is also a covering sequence of $X$. Now we prove that

$$
D_{\varphi}(\tau * \psi) \subset D_{\varphi}(\tau) \cap D_{\varphi}(\psi) \text { for every limit ordinal } \varphi<\Gamma \text {. }
$$

Proof. For $\varphi=0$ it is a consequence of the definition. Assume (4) is true for a limit ordinal $\varphi$. Thus $D_{\varphi}(\tau * \psi) \subset D_{\varphi}(\tau)$, and, using the induction, we prove that for every natural $n$

$$
D_{\varphi+2 n}(\tau * \psi) \subset D_{\varphi+n}(\tau) .
$$

Indeed, noting $\varphi+2 n=2 \varphi+2 n=2(\varphi+n)$, we see that

$$
\begin{aligned}
D_{\varphi+2(n+1)}(\tau * \psi) & \subset D_{\varphi+2 n+1}(\tau * \psi)=\operatorname{cl}\left(D_{\varphi+2 n}(\tau * \psi) \backslash Z_{\varphi+2 n}\right) \\
& =\operatorname{cl}\left(D_{\varphi+2 n}(\tau * \psi) \backslash Z_{2(\varphi+n)}\right)=\operatorname{cl}\left(D_{\varphi+2 n}(\tau * \psi) \backslash X_{\varphi+n}\right) \\
& \subset \operatorname{cl}\left(D_{\varphi+n}(\tau) \backslash X_{\varphi+n}\right)=D_{\varphi+(n+1)}(\tau) .
\end{aligned}
$$

Hence, by the definition,

$$
\begin{aligned}
D_{\varphi+\omega}(\tau * \psi) & =\bigcap_{\alpha<\varphi+\omega} D_{\alpha}(\tau * \psi)=\bigcap_{n=0}^{\infty} D_{\varphi+2 n}(\tau * \psi) \\
& \subset \bigcap_{n=0}^{\infty} D_{\varphi+n}(\tau)=D_{\varphi+\omega}(\tau) .
\end{aligned}
$$

In an analogous way we prove that $D_{\varphi+\omega}(\tau * \psi) \subset D_{\varphi+\omega}(\psi)$, thus

$$
D_{\varphi+\omega}(\tau * \psi) \subset D_{\varphi+\omega}(\tau) \cap D_{\varphi+\omega}(\psi) .
$$


Now taking into account again the intersection definition of the sets $D_{\alpha}$ for limit numbers $\alpha$, and again using the induction we get the required conclusion (4).

If $f: X \rightarrow Y$ is a surjection between compact spaces $X$ and $Y$, and $\tau=\left\{X_{\alpha}\right\}_{\alpha<\Gamma}$ is a covering sequence of $X$, then the covering sequence $\left\{f\left(X_{\alpha}\right)\right\}_{\alpha<\Gamma}$ of $Y$ will be denoted by $f(\tau)$. The proof of the following is quite similar to the proof of Lemma 17(a) of [P1], so it is omitted.

If $\tau$ is a covering sequence of a compact space $X$ and $f$ :

$X \rightarrow Y$ is a surjection, then

$$
D_{\alpha}(f(\tau)) \subset f\left(D_{\alpha}(\tau)\right) \text { for every } \alpha<\Gamma \text {. }
$$

Let a class $\mathscr{F}$ of compact spaces be invariant with respect to continuous mappings, i.e., we assume that each continuous image of any member of $\mathscr{F}$ belongs to $\mathscr{F}$. Denote by $\mathscr{F}_{1}$ the class of all compact spaces being countable unions of their compact subsets belonging to $\mathscr{F}$. Then, of course, each element of $\mathscr{F}_{1}$ admits covering sequences composed of elements of $\mathscr{F}$. Let $X \in \mathscr{F}_{1}$. Fix a limit ordinal $\Gamma$ with cardinality greater than card $X$. Further, put

$$
\begin{aligned}
& S(X)=\left\{\tau: \tau=\left\{X_{\alpha}\right\}_{\alpha<\Gamma} \text { is a covering sequence of } X\right. \text { with } \\
& \left.\qquad X_{\alpha} \in \mathscr{F} \text { for every } \alpha<\Gamma\right\} .
\end{aligned}
$$

Then for every $\alpha<\Gamma$ we put

$$
W_{\alpha}(X, \mathscr{F})=\bigcap\left\{D_{\alpha}(\tau): \tau \in S(X)\right\} .
$$

We need the following two properties of these sets.

$$
\begin{aligned}
& \text { There is the greatest limit number } \varphi_{0}<\Gamma \text { such that } \\
& W_{\varphi_{0}}(X, \mathscr{F}) \neq \varnothing \text {. }
\end{aligned}
$$

In fact, the sets $W_{\alpha}(X, \mathscr{F})$ form a decreasing sequence of compact subsets of $X$ with some $W_{\alpha}(X, \mathscr{F})=\varnothing$ (see (3)). By the definitions we have $W_{\varphi}(X, \mathscr{F})=$ $\bigcap\left\{W_{\alpha}(X, \mathscr{F}): \alpha<\varphi\right\}$ for limit ordinals $\varphi$. Thus the number $\alpha_{0}=\min \{\alpha$ : $\left.W_{\alpha}(X, \mathscr{F})=\varnothing\right\}$ is nonlimit, so $\alpha_{0}=\varphi_{0}+n$, where $\varphi_{0}$ is limit and $n>0$ is natural. The number $\varphi_{0}$ satisfies the required condition.

There is a covering sequence $\tau \in S(X)$ with $\lambda(\tau)=\varphi_{0}+n$

for some natural $n>0$, where $\varphi_{0}$ is as in (6).

Indeed, since $\varnothing=W_{\varphi_{0}+\omega}(X, \mathscr{F})=\bigcap\left\{D_{\varphi_{0}+\omega}(\tau): \tau \in S(X)\right\}$ and since the sets $D_{\alpha}(\tau)$ are compact, there is a finite system $\tau_{1}, \ldots, \tau_{m} \in S(X)$ with $\bigcap\left\{D_{\varphi_{0}+\omega}\left(\tau_{i}\right)\right.$ : $i \in\{1, \ldots, m\}\}=\varnothing$. Put $\tau=\left(\cdots\left(\tau_{1} * \tau_{2}\right) * \cdots * \tau_{m-1}\right) * \tau_{m}$. Then $D_{\varphi_{0}+\omega}(\tau) \subset$ $\bigcap\left\{D_{\varphi_{0}, \omega}\left(\tau_{i}\right): i \in\{1, \ldots, m\}\right\}=\varnothing$ by (4). Hence $\lambda(\tau) \leqslant \varphi_{0}+\omega$. But $\lambda(\tau)$ is always nonlimit (it may be observed by the definition), and $\varnothing \neq W_{\varphi_{0}}(X, \mathscr{F}) \subset$ $D_{\varphi_{11}}(\tau)$, thus $\lambda(\tau)=\varphi_{0}+n$ for some natural $n>0$.

2. Lemma. If $X \in \mathscr{F}_{1}$ and $f: X \rightarrow Y$ is a surjection, then for each limit ordinal $\varphi<\Gamma$ we have

$$
W_{\varphi}(Y, \mathscr{F}) \subset f\left(W_{\varphi}(X, \mathscr{F})\right) .
$$

Proof. Let $y \notin f\left(W_{\varphi}(X, \mathscr{F})\right)$, i.e.,

$$
\begin{aligned}
\varnothing & =f^{-1}(y) \cap W_{\varphi}(X, \mathscr{F})=f^{-1}(y) \cap \bigcap\left\{D_{\varphi}(\tau): \tau \in S(X)\right\} \\
& =\bigcap\left\{f^{-1}(y) \cap D_{\varphi}(\tau): \tau \in S(X)\right\} .
\end{aligned}
$$


Since all of the sets $f^{-1}(y) \cap D_{\varphi}(\tau)$ are closed subsets of the compact space $X$, there is a finite system $\tau_{1}, \ldots, \tau_{m} \in S(X)$ such that $\bigcap\left\{f^{-1}(y) \cap D_{\varphi}\left(\tau_{i}\right): i \in\{1, \ldots, m\}\right\}$ $=\varnothing$. Put $\tau=\left(\cdots\left(\tau_{1} * \tau_{2}\right) * \cdots * \tau_{m-1}\right) * \tau_{m}$. Then $\tau \in S(X)$, and by (4) we get

$$
f^{-1}(y) \cap D_{\varphi}(\tau) \subset f^{-1}(y) \cap D_{\varphi}\left(\tau_{1}\right) \cap \cdots \cap D_{\varphi}\left(\tau_{m}\right)=\varnothing,
$$

hence $y \notin f\left(D_{\varphi}(\tau)\right)$. By (5) and by the definition of $W_{\varphi}(Y, \mathscr{F})$, noting that $f(\tau) \in S(Y)$, we see $W_{\varphi}(Y, \mathscr{F}) \subset D_{\varphi}(f(\tau)) \subset f\left(D_{\varphi}(\tau)\right)$. Thus $y \notin W_{\varphi}(Y, \mathscr{F})$, which completes the proof.

The following lemma plays a crucial role in the proof of Theorem 1.

3. LEMMA. If $C_{1}, C_{2}, \ldots$ is a countable sequence of compact subsets of a compact space $X$ such that $X=C_{1} \cup C_{2} \cup \cdots$, then there is a compact nonempty subset $F$ of $X$ such that

(1) $F \subset f(F)$ for every surjection $f: X \rightarrow X$, and

(2) there are a finite sequence $C_{i_{1}}, \ldots, C_{i_{n}}$ of sets and a finite sequence of mappings $f_{k}: C_{i_{k}} \rightarrow X$ for $k \in\{1, \ldots, n\}$, such that $F \subset f_{1}\left(C_{i_{1}}\right) \cup \cdots \cup f_{n}\left(C_{i_{n}}\right)$.

Proof. Let $\mathscr{F}$ be the class of all continuous images of the sets $C_{1}, C_{2}, \ldots$ Then, of course, $\mathscr{F}$ is invariant with respect to continuous mappings and $X \in \mathscr{F}_{1}$. Put $F=W_{\varphi_{0}}(X, \mathscr{F})$, where $\varphi_{0}$ is the number guaranteed by (6). Then by Lemma 2 we get $F \subset f(F)$ for each surjection $f: X \rightarrow X$. Let $\tau=\left\{X_{\alpha}\right\}_{\alpha<\Gamma}$ be a covering sequence guaranteed by (7). Then since $D_{\varphi_{0}+n}(\tau)=\varnothing$ we have

$$
F=W_{\varphi_{0}}(X, \mathscr{F}) \subset D_{\varphi_{0}}(\tau) \subset X_{\varphi_{0}} \cup X_{\varphi_{0}+1} \cup \cdots \cup X_{\varphi_{0}+(n-1)},
$$

and $X_{\varphi_{0}+i} \in \mathscr{F}$ for every $i \in\{0,1, \ldots, n-1\}$, thus the sets $X_{\varphi_{0}+i}$ are continuous images of some sets $C_{k}$, which completes the proof.

Proof of Theorem 1. Let $F \subset X$ be a set guaranteed by Lemma 3 for $C_{i}=\operatorname{cl} A_{i}$. By (2) of Lemma 3 the set $F$ may be covered by finitely many subcontinua of $X$ each containing a dense $\mathscr{K}$-connected subset. Let $F_{1}, \ldots, F_{m}$ be such sets with an additional assumption that $m$ is the minimum number. Without loss of generality we may assume that $F_{i}=\mathrm{cl} B_{i}$ for some $\mathscr{K}$-components $B_{i}$ of $X$ for $i \in\{1, \ldots, m\}$.

Let $\mathscr{A}$ be the family of all $\mathscr{K}$-components of $X$.

For every $A \in \mathscr{A}$ we have $\operatorname{cl} A \cap F \neq \varnothing$.

In fact, consider any surjection $f: X \rightarrow X$ sending a point of $B_{1}$ to a point of $A$. Then $f\left(B_{1}\right) \subset A$ and $f\left(F_{1}\right)=f\left(\operatorname{cl} B_{1}\right) \subset \operatorname{cl} A$. If $\operatorname{cl} A \cap F$ were empty, the union $f\left(F_{2}\right) \cup \cdots \cup f\left(F_{m}\right)$ of $m-1$ continua with dense $\mathscr{K}$-connected subsets would contain $F$ (since $F \subset f(F) \subset f\left(F_{1}\right) \cup \cdots \cup f\left(F_{m}\right)$ and $f\left(F_{1}\right) \cap F \subset \operatorname{cl} A \cap F=$ $\varnothing)$, contrary to the assumption on $m$.

Put $\mathscr{A}_{i}=\left\{A \in \mathscr{A}: \operatorname{cl} A \cap F_{i} \neq \varnothing\right\}$ and $G_{i}=\operatorname{cl}\left(\cup \mathscr{A}_{i}\right)$ for $i \in\{1, \ldots, m\}$. We prove that

$$
G_{i} \cap G_{j}=\varnothing \quad \text { for } i \neq j, i, j \in\{1, \ldots, m\} .
$$

Suppose $x \in G_{i} \cap G_{j}$ with $j>i$. Let $U$ be the nonempty interior of a $\mathscr{K}$-component $B_{m+1}$ of $X$ and let $f: X \rightarrow X$ be a surjection sending $x$ to a point $y \in U$. Thus $f(A), f(B) \subset B_{m+1}$ for some $A \in \mathscr{A}_{i}$ and $B \in \mathscr{A}_{j}$, and $\operatorname{cl} B_{m+1} \cap f\left(F_{i}\right) \neq \varnothing \neq$ cl $B_{m+1} \cap f\left(F_{j}\right)$. Let a surjection $g: X \rightarrow X$ send a point $p \in \operatorname{cl} B_{m+1} \cap f\left(F_{i}\right)$ to $y$. 
Then $g\left(B_{m+1}\right), \quad g f\left(B_{i}\right) \subset B_{m+1}$ and $\mathrm{cl} B_{m+1} \cap g f\left(F_{j}\right) \neq \varnothing$. Let a surjection $h$ : $X \rightarrow X$ send a point $q \in \operatorname{cl} B_{m+1} \cap g f\left(F_{j}\right)$ to $y$. Then $h\left(B_{m+1}\right), h g f\left(B_{j}\right) \subset B_{m+1}$. Therefore

$$
\begin{aligned}
F \subset & h g f(F) \subset h g f\left(F_{1}\right) \cup \cdots \cup h g f\left(F_{m}\right) \\
\subset & h g f\left(F_{1}\right) \cup \cdots \cup h g f\left(F_{i-1}\right) \cup h g f\left(F_{i+1}\right) \cup \cdots \cup h g f\left(F_{j-1}\right) \\
& \cup h g f\left(F_{j+1}\right) \cup \cdots \cup h g f\left(F_{m}\right) \cup \operatorname{cl} B_{m+1} .
\end{aligned}
$$

Thus $m-1$ sets with dense $\mathscr{K}$-connected subsets cover $F$, contrary to the assumption on $m$.

The statement (9), by (8) and by the connectedness of $X$, implies

$$
m=1 \text {. }
$$

For every $A \in \mathscr{A}$ we have $F \subset \operatorname{cl} A$.

For, let a surjection $g: X \rightarrow X$ send a point of $B_{1}$ to a point of $A$. Then $F \subset g(F) \subset g\left(\operatorname{cl} B_{1}\right)=\operatorname{cl} g\left(B_{1}\right) \subset \operatorname{cl} A$.

$$
\text { For every } A \in \mathscr{A} \text { we have } \operatorname{cl} A=X \text {. }
$$

Indeed, for a given point $x \in X$ let a surjection $f: X \rightarrow X$ send a point $y \in F$ to $x$, and let $B \in \mathscr{A}$ be a $\mathscr{K}$-component of $X$ such that $f(B) \subset A$. Then $y \in \operatorname{cl} B$ by (11). Therefore $x=f(y) \in f(\operatorname{cl} B)=\operatorname{cl} f(B) \subset \operatorname{cl} A$. Thus we have (12).

To make the proof of Theorem 1 complete, note that since every $\mathscr{K}$-component of $X$ is dense in $X$ and one $\mathscr{K}$-component has nonempty interior, this $\mathscr{K}$-component is the only one.

Applications and questions. As an immediate consequence of Theorem 1 and of the Baire theorem we have the following corollary.

4. COROLlARY. If a continuously homogeneous continuum is the countable union of arcwise connected ( $\mathscr{K}$-connected) continua, then it is arcwise connected ( $K$-connected).

5. THEOREM. If a continuously homogeneous nondegenerate metric continuum $X$ contains no simple triod and it has only countably many arc components, then $X$ is either an arc or a simple closed curve.

Proof. Let $A$ be an arc component of $X$. Then one of the following statements is true:

(1) $A$ is degenerate,

(2) $A$ is a simple closed curve,

(3) $A$ is nondegenerate and it contains no simple closed curve.

In fact, note that if $A$ contains a simple closed curve, then $A$ itself is a simple closed curve (otherwise $A$ would contain a simple triod).

In case (3), since $A$ contains no simple closed curve, for all points $a, b \in A$ with $a \neq b$ there is only one arc $a b$ in $A$. Further we observe that in this case one of the following is true:

(3.1) $A$ has two end points,

(3.2) $A$ has one end point,

(3.3) $A$ has no end point. 
Namely, if $e_{1}, e_{2} \in A$ are distinct end points of $A$, then each point $p \in A$ belongs to $e_{1} e_{2}$. Indeed, if not, let $q$ be the first point of the arc $p e_{1}$ lying in the arc $e_{1} e_{2}$. Then $p q \cup q e_{1} \cup q e_{2}$ is a simple triod for $q \neq e_{1}$ and $q \neq e_{2}$. Thus (3.1)-(3.3) are all possibilities and in case (3.1) $A$ is an arc.

In case (3.2) let $e$ be the end point of $A$. Then we inductively construct a well-ordered sequence $\left\{A_{\alpha}\right\}$ of arcs contained in $A$ with $\cup_{\alpha} A_{\alpha}=A$. Namely,

$$
\begin{gathered}
A_{0}=e p \text { for a point } p \in A \backslash\{e\}, \\
A_{\alpha}=e q \text { for a point } q \in A \backslash \bigcup\left\{A_{\beta}: \beta<\alpha\right\}, \text { for } \alpha>0
\end{gathered}
$$

(if such $q$ exists). Let $A_{\alpha}=e x$ and $A_{\beta}=e y$ for $\alpha>\beta$. We have $x \notin e y$, thus ex $\not \subset$ ey, so ey $\subsetneq$ ex. Hence the sequence $\left\{A_{\alpha}\right\}$ is increasing, thus countable. Further, we may observe that it is a one-to-one image of the half-line.

Similarly we prove that in case (3.3) $A$ is a one-to-one image of the real line (details of this proof are left to the reader).

Each of these cases implies that $A$ is an $F_{\sigma}$-set, thus each arc component of $X$ is an $F_{\sigma}$-set. Since $X$ has countably many arc components only, by the Baire theorem, we infer that at least one of them has nonempty interior. Applying Theorem 1 we see that $X$ is arcwise connected. Thus $X$ is the only arc component satisfying either (2) or (3). Suppose it is neither an arc nor a simple closed curve. Thus it is nonlocally connected (since nondegenerate atriodic locally connected continuum is either an arc or a simple closed curve). There are two possibilities only: (3.2) and (3.3), i.e., $X$ is a one-to-one image either of the half-line or of the real line. But Krupski showed in [K1, Theorem 4, p. 352] that compact nonlocally connected one-to-one images of the half-line or of the real-line are not continuously homogeneous. This contradiction completes the proof.

J. J. Charatonik and T. Maćkowiak posed in [CM, Problem 3.11] the problem of characterizing continuously homogeneous arc-like continua. The former of the following two corollaries may be considered as a step in a way to do it. It also improves Corollary 1 of [K1, p. 354].

6. Corollary. Let $X$ be a nondegenerate continuously homogeneous metric continuum with only countably many arc components. Then the following statements are equivalent:

(a) $X$ is an arc,

(b) $X$ is arc-like,

(c) X contains neither a simple triod nor a simple closed curve.

7. Corollary. Under the same assumptions as in Corollary 6, the following statements are equivalent:

(a) $X$ is a simple closed curve,

(b) $X$ is circle-like,

(c) $X$ is not an arc and it contains no simple triod.

There are some interesting questions closely related to the subject of this paper, and also to the results of $[\mathbf{K} 1, \mathbf{K} 2, \mathbf{C G}]$. 
QUESTION 1. If a continuum $X$ is continuously homogeneous and has $\mathscr{K}$-components (arc components) $A_{1}, A_{2}, \ldots$ with $X=\operatorname{cl} A_{1} \cup \mathrm{cl} A_{2} \cup \cdots$, is each $\mathscr{K}$-component (arc component) of $X$ necessarily dense in $X$ ?

We know that each arc component of a continuously homogeneous continuum $X$ with finitely many arc components is dense in $X$ (see Theorem 3 of [CG]).

QUESTION 2. If a continuously homogeneous continuum $X$ has only countably many $\mathscr{K}$-components (arc components), is each $\mathscr{K}$-component (arc component) necessarily dense in $X$ ?

Question 3. Under the same conditions as in Question 2, is $X$ necessarily $\mathscr{K}$-connected (arcwise connected)?

QUESTION 4. What about an answer to Question 3 if we additionally assume that $X$ has only a finite number of $\mathscr{K}$-components (arc components)?

\section{REFERENCES}

[C] J. J. Charatonik, A characterization of the pseudo-arc, Bull. Acad. Polon. Sci. Sér. Sci. Math. Astronom. Phys. 26 (1978), 901-903.

[CM] J. J. Charatonik and T. Maćkowiak, Confluent and related mappings on arc-like continua-an application to homogeneity, Topology and Appl. 23 (1986), 29-39.

[CG] W. J. Charatonik and Z. Garncarek, Some remarks on continuously homogeneous continua, Bull. Polish Acad. Sci. Math. 32 (1984), 339-342.

[H1] C. L. Hagopian, Mapping theorems for plane continua, Topology Proc. 3 (1978), 117-122.

[H2] _. Planar $\lambda$ connected continua, Proc. Amer. Math. Soc. 39 (1973), 190-194.

$[\mathrm{H3}] \_, \lambda$ connected plane continua, Trans. Amer. Math. Soc. 191 (1974), 277-287.

[K1] P. Krupski, Continua which are homogeneous with respect to continuity, Houston J. Math. 5 (1979), $345-356$.

[K2] Continuous homogeneity of continua, Proceedings of the International Conference on Geometric Topology, PWN-Polish Scientific Publishers, Warszawa, 1980, pp. 269-272.

[KM] K. Kuratowski and A. Mostowski, Set theory, PWN-Polish Scientific Publishers, Warszawa, 1967.

[L] A. Lelek, On weakly chainable continua, Fund. Math. 51 (1962-63), 271-282.

[P1] J. R. Prajs, On continuous mappings onto some Cartesian products of compacta, Houston J. Math. (to appear).

[P2] __ Some invariants under perfect mappings and their applications to continua (to appear).

Institute of Mathematics, Opole Pedagogical University, ul. Oleska 48, 45-951 Opole, POI.ANI) 